\title{
Norepinephrine intravenous prophylactic bolus versus rescue bolus to prevent and treat maternal hypotension after combined spinal and epidural anesthesia during cesarean delivery: a sequential dose-finding study
}

\author{
Tao Xu ${ }^{1,2,3}$, Jing Zheng ${ }^{1,2,3}$, Xiao-Hu An ${ }^{1,2,3}$, Zi-Feng Xu ${ }^{1,2,3}$, Fang Wang ${ }^{4}$ \\ ${ }^{1}$ Department of Anesthesiology, the International Peace Maternity and Child Health Hospital, School of Medicine, Shanghai Jiao Tong University, \\ Shanghai 200030, China; ${ }^{2}$ Shanghai Key Laboratory of Embryo Original Diseases, Shanghai 20030, China; ${ }^{3}$ Shanghai Municipal Key Clinical \\ Specialty, Shanghai 20030, China; ${ }^{4}$ Department of Biostatistics, St. Jude Children's Research Hospital, Memphis, TN, USA \\ Contributions: (I) Conception and design: XH An, ZF Xu; (II) Administrative support: XH An, ZF Xu; (III) Provision of study materials or patients: J \\ Zheng; (IV) Collection and assembly of data: T Xu, J Zheng, F Wang; (V) Data analysis and interpretation: T Xu, J Zheng, F Wang; (VI) Manuscript \\ writing: All authors; (VII) Final approval of manuscript: All authors. \\ Correspondence to: Jing Zheng, MD. Department of Anesthesiology, the International Peace Maternity and Child Health Hospital, School of \\ Medicine, Shanghai Jiao Tong University, Henshan Road 910, Shanghai 200030, China. Email: Sukura5203yy@sjtu.edu.cn.
}

Background: As a relatively new drug in obstetrical anesthesia, norepinephrine is less likely to induce bradycardia and decrease cardiac output, which makes it a potential alternative to phenylephrine. The purpose of this study was to determine the optimal norepinephrine bolus dose needed to either prevent or reverse hypotension after the use of combined spinal and epidural (CSE) anesthesia in $90 \%$ of women during elective cesarean delivery (CD).

Methods: Eighty women undergoing elective CD were randomly allocated into either a prophylactic group or a rescue group in this dose finding study. If the women's systolic blood pressure (SBP) was maintained above $80 \%$ of their baseline, the next patient had an $8 / 9^{\text {th }}$ chance of receiving the same dose or a $1 / 9^{\text {th }}$ chance of receiving a lower dose. If the patient's SBP was not maintained, a higher dose was used for next patient. The primary outcome was the successful use of the norepinephrine bolus dose to maintain SBP above $80 \%$ of the baseline until after delivery. Secondary outcomes included nausea, vomiting, breathlessness, dizziness, hypertension, bradycardia due to hypotension and supplemental use of atropine and norepinephrine, upper sensory level of anesthesia, umbilical vein (UV) blood gases, and 1- and 5-minute Apgar scores. The 90\% effective dose $\left(\mathrm{ED}_{90}\right)$ and $95 \%$ confidence interval $(95 \% \mathrm{CI})$ were estimated using isotonic regression methods.

Results: The estimated $\mathrm{ED}_{90}$ of the norepinephrine prophylactic bolus was $10.85 \mu \mathrm{g}(95 \%$ CI, 9.20$11.67 \mu \mathrm{g})$ and that of the norepinephrine rescue bolus was $12.3 \mu \mathrm{g}(95 \% \mathrm{CI}, 10.0-12.8 \mu \mathrm{g}) \mathrm{using}$ isotonic regression methods.

Conclusions: For norepinephrine, either a prophylactic bolus dose of $11 \mu \mathrm{g}$ or a rescue bolus dose of $12 \mu \mathrm{g}$ was recommended for clinical practices.

Keywords: Norepinephrine; prophylactic bolus; rescue bolus; $90 \%$ effective dose $\left(\mathrm{ED}_{90}\right)$; maternal hypotension; cesarean delivery $(\mathrm{CD})$

Submitted May 15, 2019. Accepted for publication Aug 08, 2019.

doi: $10.21037 / \mathrm{atm} .2019 .08 .50$

View this article at: http://dx.doi.org/10.21037/atm.2019.08.50 


\section{Introduction}

Norepinephrine, as a relatively new drug in obstetric anesthesia, has $\alpha$-adrenergic and weak $\beta$-adrenergic effects. Compared with phenylephrine, it is less likely to induce bradycardia and decreased cardiac output (1-3), which makes it a potential alternative to phenylephrine.

Prophylactic use of vasopressors is increasingly considered to be a standard measure during spinal anesthesia (SA) for cesarean delivery (CD) (4-7). However, few developing countries incorporate prophylactic vasopressors into their delivery management guidelines (8).

This prospective, double-blind, randomized, sequential dose finding study aims to identify the $90 \%$ effective dose $\left(\mathrm{ED}_{90}\right)$ value of norepinephrine for both prophylactic and rescue bolus doses by using the biased coin up-and-down (BCUD) design.

\section{Methods}

\section{Materials and methods}

After obtaining approval from the Research Ethics Committee of the International Peace Maternity and Child Health Hospital and registering at http://www.chictr.org.cn (INR-1800016213), we began a prospective, double-blind, randomized, sequential dose-finding study, using a BCUD design to estimate $\mathrm{ED}_{90}$. A total of 109 women aged 1840 years, with a full-term ( $>37$ weeks' gestation) singleton pregnancy, ASA score II, and undergoing an elective CD were recruited during the study period from January 2018 to August 2018. After providing signed informed consent, the women were randomly allocated into two groups according to the randomized numbers generated by a research assistant using SPSS for Windows version 18.0 (SPSS Inc., Chicago, IL, USA): the prophylactic bolus group (Group P) and the rescue bolus group (Group R). Exclusion criteria for the study were as follows: allergy to norepinephrine, preexisting or pregnancyinduced hypertension, preeclampsia, cardiovascular or cerebrovascular disease, fetal distress or fetal abnormalities, multiple gestations, patient refusal, and emergent CD.

Patients were instructed to fast after midnight on the day of surgery. After entering the operating room, an intravenous (IV) line was established with an $18-\mathrm{G}$ IV catheter in the left forearm, and lactated Ringer's solution was infused at a rate of $1 \mathrm{~mL} / \mathrm{min}$ to maintain the vein open. Routine monitoring, including electrocardiography, noninvasive blood pressure, and pulse oximetry, was performed continuously. Systolic blood pressure (SBP), mean arterial pressure (MAP), heart rate (HR), and pulse oximetry were assessed once a minute (min). The first two resting SBP, MAP, and HR measurements in a supine position were recorded, and their average values were treated as the baseline pressure and HR. If the baseline SBP was above $140 \mathrm{mmHg}$, the patient was excluded from the study due to hypertension. The combined spinal and epidural (CSE) anesthesia was performed by experienced anesthesiologists. The CSE puncture was performed routinely at the level of L3-4 with the patient in the right lateral decubitus position. A $17 \mathrm{G}$ Tuohy needle was used to perform the epidural puncture with a paramedian approach. After identifying entrance into the epidural space, a 27-G Whitacre needle was then inserted through the epidural needle. As soon as the cerebrospinal fluid was detected, $10.5 \mathrm{mg}$ (9) of $0.75 \%$ isobaric ropivacaine was injected through the Whitacre needle. After injecting the ropivacaine, an epidural catheter was inserted into the epidural space by advancing it $3 \mathrm{~cm}$ through the Tuohy needle. After the patient was moved to a supine position with left uterine displacement created by placing a wedge under the right hip, $5 \mathrm{~mL}$ of $2 \%$ lidocaine was administered through the epidural catheter. In addition, an infusion of $1 \mathrm{~mL} / \mathrm{kg} / \mathrm{min}$ lactated Ringer's solution was administered until delivery. Another $5 \mathrm{~mL}$ of $2 \%$ lidocaine was administered through the epidural catheter $3 \mathrm{~min}$ after the initial dose if the patient could still feel the cold sensation of ice being placed on the skin below the level of T6. Patients who still had a sensory response to ice below the level of T6 at the beginning of surgery were excluded from the study. Immediately after delivery, $1 \mathrm{~mL}$ of umbilical vein (UV) blood was collected by the obstetrician, and blood gas assessments were performed using a blood gas analyzer (iSTAT1 Analyzer MN:300-G, Abbott Point of Care Inc., USA) with an iSTAT CG7+ test cartridge.

In Group P, a 5-mL prophylactic dose of norepinephrine in a $5-\mathrm{mL}$ syringe prepared by a research assistant was administered via the IV catheter immediately after the patient was moved to a supine position. An additional $5-\mathrm{mL}$ syringe with a rescue bolus of $6 \mu \mathrm{g}$ of norepinephrine was prepared by the research assistant, and the patient received the rescue norepinephrine bolus of $6 \mu \mathrm{g}$ immediately after the first episode of hypotension was detected. In Group R, $5 \mathrm{~mL}$ of normal saline in a $5-\mathrm{mL}$ syringe prepared by the research assistant was administered immediately after the patient was moved to a supine position. Then, the rescue dose of norepinephrine in a $5-\mathrm{mL}$ syringe prepared by the research assistant was administered immediately after the 
first episode of hypotension was detected. The research assistant was the only person who knew which drugs and doses were in which syringes. All anesthesiologists and patients were blinded to the drugs and doses. Hypotension was defined as having a SBP $<80 \%$ of the baseline value. If the SBP of patients in either group could not be maintained at $80 \%$ of the baseline after the initial drug administration, then the additional rescue norepinephrine bolus of $6 \mu \mathrm{g}$ in a 2 -mL syringe would be administered by the anesthesiologist. To differentiate from the initial drugs, the additional rescue norepinephrine boluses of $6 \mu \mathrm{g}$ were prepared in 2-mL syringe by anesthesiologist. If bradycardia, defined as HR below 50 beats per minute (BPM), was detected, then $0.5 \mathrm{mg}$ atropine was administered to increase HR. The atropine and norepinephrine were given separately. Hypertension was defined as a SBP $>120 \%$ of the baseline value.

The bolus dose of $4 \mu \mathrm{g}$ was used for the first patient of both groups. The dose for subsequent patients was determined by the response of each previous patient. In Group P, if the SBP decreased below $80 \%$ of the baseline, the bolus dose was considered failed and the dose for the following patient was increased by $1 \mu \mathrm{g}$. If the SBP was maintained to at least $80 \%$ of the baseline, the dose used was considered a success, and the next patient was randomly assigned a dose with a $1 / 9^{\text {th }}$ chance of receiving a lower dose (decreased by $1 \mu \mathrm{g}$ ) or an $8 / 9^{\text {th }}$ chance of receiving the same dose as the previous patient. In Group R, if the SBP remained below $80 \%$ of the baseline for 2 successive 2 minutes blood pressure cycles after the bolus dose was given, or could be not maintained above $80 \%$ of the baseline until delivery, the dose was considered to have failed, and the dose for the following patient was increased by $1 \mu \mathrm{g}$. If the SBP was maintained above $80 \%$ of the baseline, the dose was considered to be a success and the next patient was randomly assigned a dose with a $1 / 9^{\text {th }}$ chance of receiving a lower dose level (decreased by $1 \mu \mathrm{g}$ ) or an $8 / 9^{\text {th }}$ chance of receiving the same dose level. This was implemented using the BCUD scheme prepared by a study statistician in Microsoft Excel 2016, and was used by the research assistant, who was the only person with access to this software, maintaining the double-blind nature of the study.

The primary outcome was the success of both the prophylactic bolus dose and the rescue bolus dose to maintain the SBP above $80 \%$ of the baseline until delivery. Secondary outcomes included both maternal and fetal observations. Maternal observations included dizziness, breathlessness, nausea (spontaneous complaints from patients recorded by anesthesia providers), vomiting, bradycardia requiring the use of atropine, use of additional rescue norepinephrine doses, hypertension, upper sensory level of anesthesia to ice cold, total co-load IV fluid, total consumption of norepinephrine. Neonatal observations included induction-delivery interval, uterine incisiondelivery interval, $\mathrm{UV}$ blood gases [including $\mathrm{pH}, \mathrm{pO}_{2}$, $\mathrm{pCO}_{2}$, base excess (BE), $\mathrm{pHCO}_{3}$, and $\mathrm{SO}_{2}$ ], neonatal weight, and Apgar scores measured at 1 and 5 min post-delivery. Maternal demographics such as age, weight, height, gestational weeks, gravida, para, baseline SBP, baseline MAP, and baseline HR were also recorded.

\section{Statistical analysis and sample size calculation}

This dose finding study based on BCUD design and simulation studies suggests that the stopping rule of enrolling 20-40 patients will provide stable estimates of the target dose for most realistic cases (10). In this study, the sample size of each group was 40 patients.

The $\mathrm{ED}_{90}$, defined as the bolus dose of norepinephrine at which the primary outcome of success was observed in $90 \%$ of the patients in the study population, was estimated by isotonic regression method $(10,11)$. The isotonic regression estimator of $\mathrm{ED}_{90}$ is the linear interpolated dose between $p^{*}(r)$ and $p^{*}(r+1): \widehat{\mathrm{U}} 3=\frac{0.9-p^{*}(r)}{p^{*}(r+1)-p^{*}(r)}(x(r+1)-x(r))+x(r)$, where $x(r)=\max \left(x(i): p^{*}(i) \leq 0.9\right)$ and $p^{*}(i)$ is the adjusted rate of the primary outcome of success at dose $x(i), i=1,2,3, \ldots$, $k$, estimated by the pooled-adjacent-violators algorithm (PAVA). Because the observed rate of $p=\{p(1), p(2), \ldots$, $p(k)\}$ might not be increased with respect to the dose level, which is the implicit assumption of a dose-finding study, the PAVA algorithm was first used in isotonic regression to obtain an increase adjusted rate $p^{*}=\left\{p^{*}(1) \leq p^{*}(2) \leq, \ldots\right.$, $\leq p(k)\}$ based on $p$. The $95 \%$ confidence interval (CI) of isotonic regression estimator of $\mathrm{ED}_{90}$ was obtained by a bias-corrected percentile method $(12,13)$ using 2,000 bootstrap replications of $\widehat{U} 3$. Each replication was obtained by drawing a bootstrap data set with sample size of 40 and BCUD design, assuming that the true dose-response rate at each dose is $p^{*}(i), i=1,2,3, \ldots, k$, estimated based on the original data. We then estimated $\widehat{\mathrm{U}} 3$, the isotonic regression estimator of $\mathrm{ED}_{90}$ based on the bootstrap data. The isotonic regression and bootstrapping were performed 


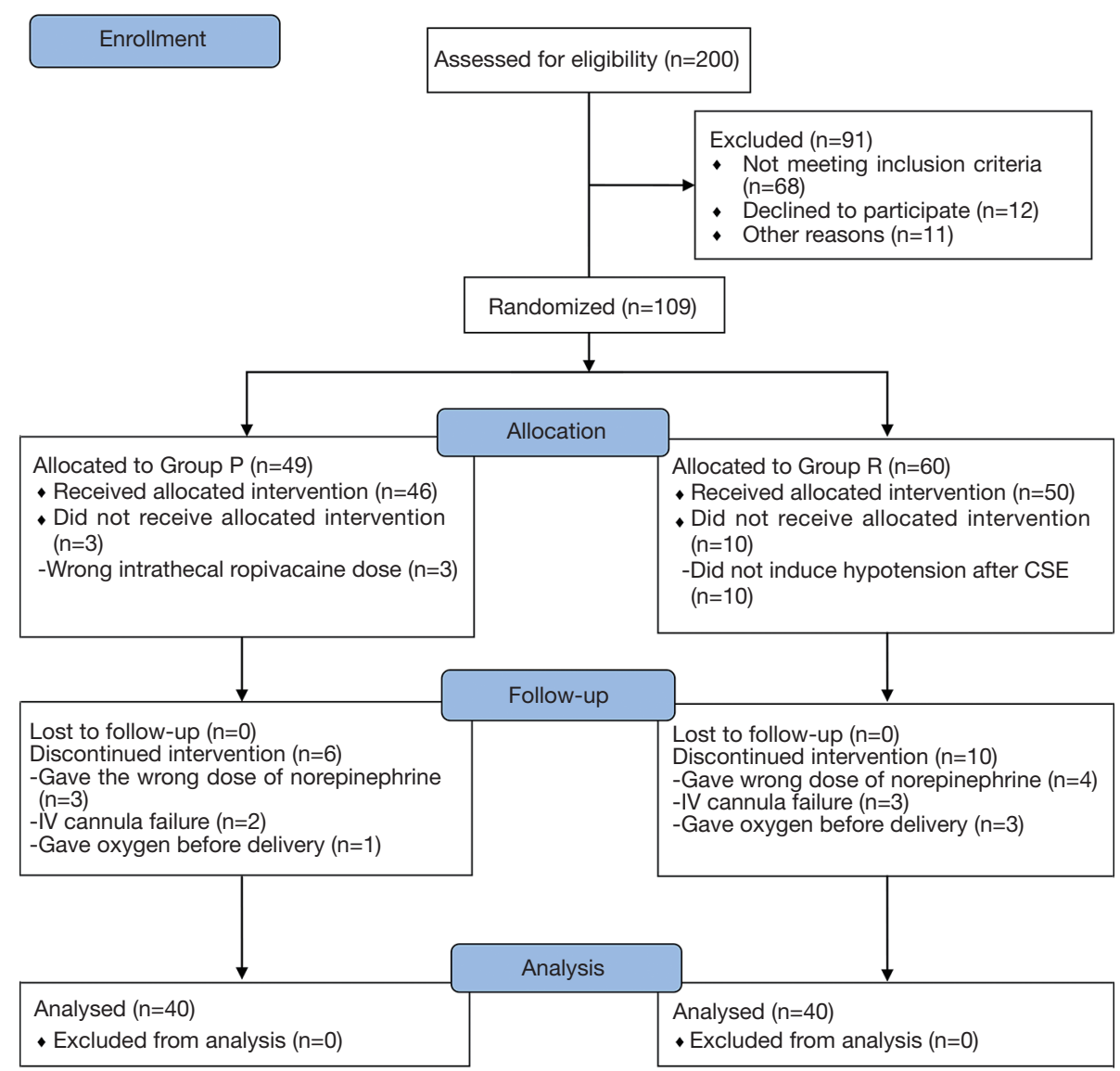

Figure 1 Flow diagram. IV, intravenous; CSE, combined spinal and epidural.

by the study statistician using $\mathrm{R}$ version 3.4.4.

The data composed of demographic variables and secondary outcomes were summarized as mean \pm standard deviations (SD), median, range, numbers, and proportions. Parametric data were analyzed with the $t$-test and nonparametric data were analyzed with the Mann-Whitney test. Comparison of proportions was performed using Chisquare test and Fisher exact tests as appropriate. Statistical comparisons were made using SPSS for Windows version 18.0 (SPSS Inc., IL, USA). Statistical significance was assumed if $\mathrm{P}<0.05$.

\section{Results}

Two hundred women who underwent elective CSE for CD were selected for the study during January 2018 to August 2018. Data used for patient recruitment are shown in the Figure 1. Forty patients from each group were included in final data analysis. Maternal characteristics are shown in Table 1.

The sequence of effective and ineffective responses at each prophylactic norepinephrine and rescue norepinephrine bolus dose for forty successive patients are shown in Figures 2,3. Tables 2,3 show the observed and PAVA-adjusted response rates for each prophylactic norepinephrine and rescue norepinephrine bolus dose level, respectively. The $\mathrm{ED}_{90}$ value of norepinephrine prophylactic bolus was $10.85 \mu \mathrm{g}(95 \% \mathrm{CI}, 9.20-11.67 \mu \mathrm{g})$ and the $\mathrm{ED}_{90}$ value of norepinephrine rescue bolus was $12.3 \mu \mathrm{g}$ (95\% CI, $10.0-12.8 \mu \mathrm{g})$ as determined using isotonic regression methods.

Table 4 shows maternal outcomes. The occurrences of unpleasant symptoms, such as dizziness $(2.5 \%$ vs. $42.5 \%$, $\mathrm{P}=0.000)$, breathlessness $(7.5 \%$ vs. $70.0 \%, \mathrm{P}=0.000)$, bradycardia $(0.0 \%$ vs. $15.0 \%, \mathrm{P}=0.026)$, and nausea $(10.0 \%$ vs. $50.0 \%, \mathrm{P}=0.000)$ related to hypotension were much 
Table 1 Maternal Characteristics

\begin{tabular}{lccc}
\hline Characteristics & $\begin{array}{c}\text { Group P } \\
(\mathrm{n}=40)\end{array}$ & $\begin{array}{c}\text { Group R } \\
(\mathrm{n}=40)\end{array}$ & P value \\
\hline Age, years & $32.1 \pm 3.5$ & $33.5 \pm 4.1$ & 0.108 \\
Weight, kg & $69.7 \pm 7.4$ & $70.7 \pm 8.7$ & 0.600 \\
Height, cm & $162.6 \pm 5.1$ & $160.7 \pm 4.7$ & 0.082 \\
Gestational weeks, weeks & $38.8 \pm 0.9$ & $38.5 \pm 0.9$ & 0.138 \\
Gravida, n & $1.85 \pm 0.9$ & $1.92 \pm 1.0$ & 0.090 \\
Para, n & $1.35 \pm 0.5$ & $1.5 \pm 0.6$ & 0.136 \\
Baseline HR, bpm & $79.5 \pm 14.1$ & $81.8 \pm 10.3$ & 0.407 \\
Baseline SBP, mmHg & $122.7 \pm 8.5$ & $119.6 \pm 10.8$ & 0.157 \\
Baseline MAP, mmHg & $87.8 \pm 8.7$ & $88.5 \pm 7.4$ & 0.680 \\
\hline
\end{tabular}

Values are mean \pm SD. HR, heart rate; SBP, systolic blood pressure; MAP, mean arterial pressure; SD, standard deviation.

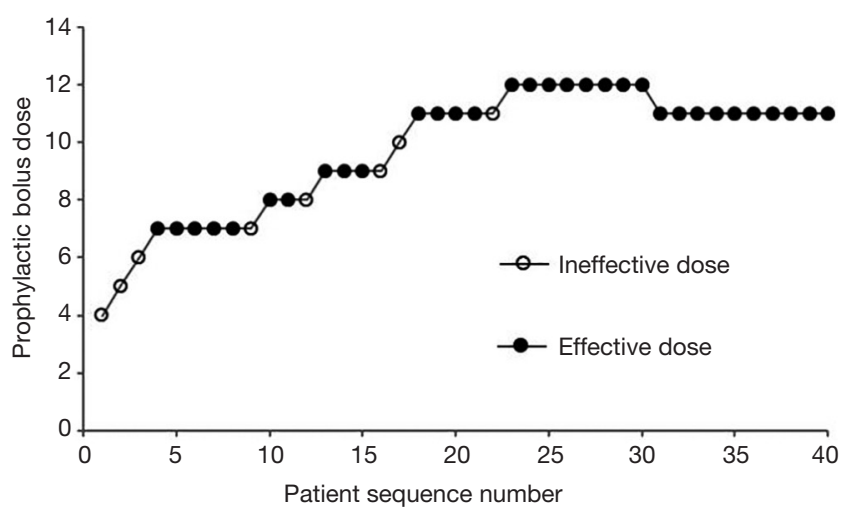

Figure 2 The patient allocation sequence and the response to the assigned dose of Group P. The patient sequence number (X-axis) is the order of patient exposures using the BCUD design. The assigned dose levels are presented on y-axis. An effective dose is denoted by a solid circle, while an ineffective one is denoted by a hollow circle. BCUD, biased coin up-and-down.

lower in Group P. The number of patients who needed atropine $(0.0 \%$ vs. $15.0 \%, \mathrm{P}=0.026)$ was less in Group P. Fewer patients in Group $\mathrm{P}$ needed additional rescue norepinephrine boluses $(5.0 \%$ vs. $25.0 \%, \mathrm{P}=0.025)$ to maintain normal blood pressure.

Table 5 shows neonatal outcomes. The results of UV blood gases were not obtained in one patient from Group $\mathrm{P}$ and four patients from Group $\mathrm{R}$ due to technical problems. The $\mathrm{PO}_{2}$ values of $\mathrm{UV}(24.5 \pm 6.7$ vs. $20.1 \pm 7.1 \mathrm{mmHg}$,

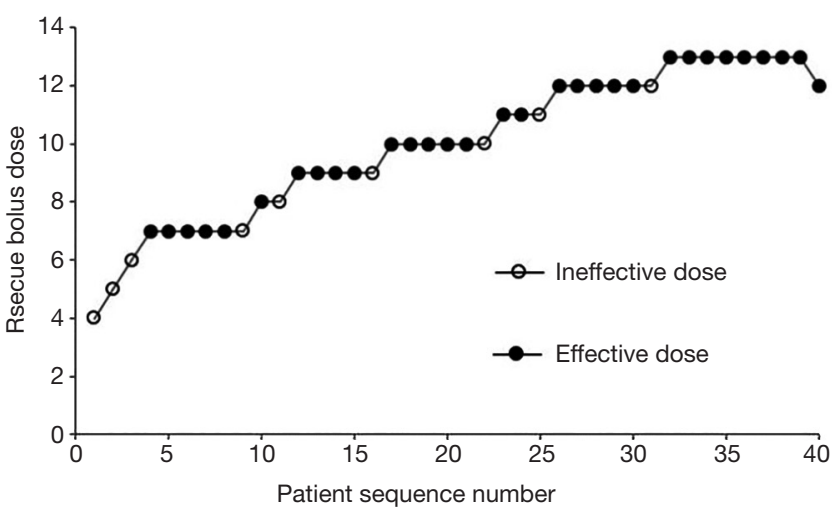

Figure 3 The patient allocation sequence and the response to the assigned dose of Group $\mathrm{R}$. The patient sequence number ( $\mathrm{X}$-axis) is the order of patient exposures using the BCUD design. The assigned dose levels are presented on y-axis. An effective dose is denoted by a solid circle, while an ineffective one is denoted by a hollow circle. BCUD, biased coin up-and-down.

Table 2 Observed and PAVA-adjusted response in Group P

\begin{tabular}{lcccc}
\hline $\begin{array}{l}\text { Assigned } \\
\text { dose, ug }\end{array}$ & $\begin{array}{c}\text { Number of } \\
\text { successes, } n\end{array}$ & $\begin{array}{c}\text { Number of } \\
\text { patients, } n\end{array}$ & $\begin{array}{c}\text { Observed } \\
\text { response } \\
\text { rate, } \%\end{array}$ & $\begin{array}{c}\text { PAVA-adjusted } \\
\text { response } \\
\text { rate, } \%\end{array}$ \\
\hline 4 & 0 & 1 & 0.00 & 0.000 \\
5 & 0 & 1 & 0.00 & 0.000 \\
6 & 0 & 1 & 0.00 & 0.000 \\
7 & 5 & 6 & 0.83 & 0.714 \\
8 & 2 & 3 & 0.67 & 0.714 \\
9 & 3 & 4 & 0.75 & 0.714 \\
10 & 0 & 1 & 0.00 & 0.714 \\
11 & 14 & 15 & 0.93 & 0.933 \\
12 & 8 & 8 & 1.00 & 1.000 \\
\hline
\end{tabular}

PAVA-adjusted response rates were estimated using the weighted isotonic regression method. PAVA, pooled-adjacentviolators algorithm.

$\mathrm{P}=0.008)$ were higher in Group $\mathrm{P}$. The $\mathrm{SO}_{2}$ values of $\mathrm{UV}$ $(41.6 \% \pm 17.2 \%$ vs. $30.2 \% \pm 13.3 \%, \mathrm{P}=0.002)$ were higher in Group P.

\section{Discussion}

In this sequential dose-finding study, the $\mathrm{ED}_{90}$ value of norepinephrine for both prophylactic and rescue bolus doses to prevent hypotension after CSE during CD were 
Table 3 Observed and PAVA-adjusted response in Group R

\begin{tabular}{llccc}
\hline $\begin{array}{l}\text { Assigned } \\
\text { dose, ug }\end{array}$ & $\begin{array}{c}\text { Number of } \\
\text { successes, } \mathrm{n}\end{array}$ & $\begin{array}{c}\text { Number of } \\
\text { patients, } \mathrm{n}\end{array}$ & $\begin{array}{c}\text { Observed } \\
\text { response } \\
\text { rate, } \%\end{array}$ & $\begin{array}{c}\text { PAVA-adjusted } \\
\text { response } \\
\text { rate, \% }\end{array}$ \\
\hline 4 & 0 & 1 & 0.00 & 0.000 \\
5 & 0 & 1 & 0.00 & 0.000 \\
6 & 0 & 1 & 0.00 & 0.000 \\
7 & 5 & 6 & 0.83 & 0.750 \\
8 & 1 & 2 & 0.50 & 0.750 \\
9 & 4 & 5 & 0.80 & 0.786 \\
10 & 5 & 6 & 0.83 & 0.786 \\
11 & 2 & 3 & 0.67 & 0.786 \\
12 & 6 & 7 & 0.86 & 0.857 \\
13 & 8 & 8 & 1.00 & 1.000 \\
\hline
\end{tabular}

PAVA-adjusted response rates were estimated using the weighted isotonic regression method. PAVA, pooled-adjacentviolators algorithm.

Table 4 Maternal outcomes

\begin{tabular}{lccc}
\hline Outcomes & $\begin{array}{c}\text { Group P } \\
(\mathrm{n}=40)\end{array}$ & $\begin{array}{c}\text { Group R } \\
(\mathrm{n}=40)\end{array}$ & P value \\
\hline Hypertension & $0(0.0)$ & $0(0.0)$ & 1.000 \\
Bradycardia & $0(0.0)$ & $6(15.0)$ & 0.026 \\
Dizziness & $1(2.5)$ & $17(42.5)$ & 0.000 \\
Atropine use & $0(0.0)$ & $6(15.0)$ & 0.026 \\
Breathlessness & $3(7.5)$ & $28(70.0)$ & 0.000 \\
Nausea & $4(10.0)$ & $20(50.0)$ & 0.000 \\
Vomiting & $0(0.0)$ & $1(2.5)$ & 1.000 \\
Additional norepinephrine & $2(5.0)$ & $10(25.0)$ & 0.025 \\
bolus use & & & \\
Upper sensory level & $\mathrm{T} 3[\mathrm{~T} 1-\mathrm{T} 6]$ & $\mathrm{T} 3[\mathrm{~T} 1-\mathrm{T} 6]$ & 0.131 \\
Total co-load IV fluid, $\mathrm{mL}$ & $830 \pm 194$ & $849 \pm 221$ & 0.679 \\
Total norepinephrine & $11[7-17]$ & $12[7-20]$ & 0.267 \\
consumption, ug & & & \\
\hline
\end{tabular}

Values are mean $\pm \mathrm{SD}, \mathrm{n}(\%)$ or median [range]. IV, intravenous; $\mathrm{SD}$, standard deviation.

estimated. The results showed that the $\mathrm{ED}_{90}$ value of prophylactic norepinephrine bolus was $10.85 \mu \mathrm{g}$ (95\% CI, 9.20-11.67 $\mu \mathrm{g}$ ) and the $\mathrm{ED}_{90}$ value of rescue norepinephrine bolus was $12.3 \mu \mathrm{g}$ (95\% CI, 10.0-12.8 $\mu \mathrm{g}$ ) using isotonic regression methods. The occurrences of unpleasant symptoms, such as dizziness, breathlessness, bradycardia, and hypotension related nausea were much lower in Group P. Fewer patients needed atropine or additional norepinephrine boluses in Group P. The $\mathrm{PO}_{2}$ and $\mathrm{SO}_{2}$ values of the UV blood gases were higher in Group P.

The latest study by Onwochei et al. (14) showed the $\mathrm{ED}_{90}$ for prophylactic intermittent bolus doses of norepinephrine, aimed at maintaining patients' blood pressure at their baseline, was $5.8 \mu \mathrm{g}$ (95\% CI, 5.01-6.59 $\mu \mathrm{g})$. Intermittent use of this bolus dose was proven to be safe and effective to prevent hypotension after SA. According to Onwochei's (14) and Ngan Kee's study (15), a dose of $4 \mu \mathrm{g}$ was cautiously chosen for the first patient of this sequential study. In Onwochei's study, the total consumption of norepinephrine ranged from 6 to $78 \mu \mathrm{g}$, which was much higher when compared to the current study $(11 \mu \mathrm{g}, 7-17 \mu \mathrm{g})$. This may partly due to the difference in the study methods. In the Onwochei's study, the target for the patients' blood pressure was set as the baseline and the patients were intermittently given a bolus dose of norepinephrine when BP fell below the baseline. To some extent, their treatment of hypotension after SA more approximated a rescue therapy, although it aimed to estimate the $\mathrm{ED}_{90}$ dose of prophylactic norepinephrine. In this study, a real sense of the $\mathrm{ED}_{90}$ of prophylactic norepinephrine was estimated, which caused the increased $\mathrm{ED}_{90}$ value and decreased the total consumption of norepinephrine.

Ngan Kee et al. (15) designed a dose-response study to estimate $\mathrm{ED}_{50}$ and $\mathrm{ED}_{90}$ of norepinephrine to treat the first episode of hypotension after SA. The resulting $\mathrm{ED}_{90}$ dose of norepinephrine was $18 \mu \mathrm{g}$, which was higher than that in the current study $(12.3 \mu \mathrm{g})$. The target $\mathrm{BP}$ was also set to $80 \%$ of the baseline, the same as in the current study. However, the patients in that study were divided into six different dose groups (4, 5, 6, 8, 10, and $12 \mu \mathrm{g})$, and adopted a dose-response method, which was different from the current study. More importantly, the response rate of the 12 $\mu \mathrm{g}$ group was above $95 \%$, which was similar to the result of the current study $(12.3 \mu \mathrm{g})$. In the dose-response study, the estimated $\mathrm{ED}_{90}$ dose of norepinephrine was $18 \mu \mathrm{g}$ and this result was based on their special statistic method; however, the dose groups above $18 \mu \mathrm{g}$ were absent from that study.

It should be noted that the spinal drugs and doses were not same in both studies above $(13,14)$, although the same anesthesia method for the patient of SA was adopted. The CSE was adopted in the current study, which was different from the studies above. The difference of the rate and 
Table 5 Neonatal outcomes

\begin{tabular}{|c|c|c|c|}
\hline Outcomes & $\begin{array}{l}\text { Group P } \\
(n=40)\end{array}$ & $\begin{array}{l}\text { Group R } \\
(n=40)\end{array}$ & $P$ value \\
\hline $\begin{array}{l}\text { Induction-delivery interval, } \\
\text { minutes }\end{array}$ & $12.0 \pm 2.9$ & $12.0 \pm 2.6$ & 1.000 \\
\hline $\begin{array}{l}\text { Uterine incision-delivery } \\
\text { interval, seconds }\end{array}$ & $89 \pm 59$ & $99 \pm 74$ & 0.511 \\
\hline UV, pH & $7.36 \pm 0.03$ & $7.36 \pm 0.11$ & 0.722 \\
\hline $\mathrm{PO}_{2}, \mathrm{mmHg}$ & $24.5 \pm 6.7$ & $20.1 \pm 7.1$ & 0.008 \\
\hline $\mathrm{PCO}_{2}, \mathrm{mmHg}$ & $41.1 \pm 5.8$ & $43.7 \pm 6.5$ & 0.079 \\
\hline $\mathrm{BE}, \mathrm{mEq} / \mathrm{L}$ & $-2.0 \pm 1.6$ & $-1.9 \pm 1.6$ & 0.816 \\
\hline $\mathrm{SO}_{2}, \%$ & $41.6 \pm 17.2$ & $30.2 \pm 13.3$ & 0.002 \\
\hline $\mathrm{HCO}_{3}, \mathrm{mmHg}$ & $23.3 \pm 1.0$ & $24.1 \pm 2.1$ & 0.116 \\
\hline Neonatal weight, g & $3,438 \pm 507$ & $3,367 \pm 379$ & 0.483 \\
\hline Apgar Scores at $1 \mathrm{~min}$ & $10[10-10]$ & $10[8-10]$ & 0.494 \\
\hline Apgar Scores at $5 \mathrm{~min}$ & $10[10-10]$ & $10[10-10]$ & 1.000 \\
\hline
\end{tabular}

Values are mean \pm SD or median [range]. UV, umbilical vein; BE, base excess; SD, standard deviation.

degree of hypotension in patients was difficult to evaluate due to the prophylactic drug use method, but the median upper anesthetic levels of patients were close (T2 to T4) between the current study, Onwochei's study, and Ngan Kee's study. The increasing incidence of hypotension after SA for CD with higher analgesic levels above T4 was proven by several studies $(16,17)$; therefore, we can reasonably infer that the hypotension rates were not much different among three studies.

In the current study, the prophylactic use of a small bolus of norepinephrine was employed in an attempt to prevent the hypotension after SA during CD. It may have more advantages in reduction of unpleasant symptoms related to hypotension and the total consumption of rescue dose of vasopressors, increasing oxygen supplementation for fetuses.

The $\mathrm{ED}_{90}$ dose of norepinephrine in the current study had been proven to be safe by the studies of Onwochei and Ngan Kee. Side effects such as maternal tissue ischemia on hands and forearms, severe maternal hypertension, severe maternal tachycardia, and adverse effect to fetuses and neonates were absent. Only one patient in Group R and two patients in Group P encountered hypertension soon after norepinephrine was given, and all patients' blood pressures came back to baseline in $3 \mathrm{~min}$. None of them complained about hypertension related side effects such as headaches and nausea. Furthermore, all three patients were more tolerant to anesthesia and had their upper sensory level below T6; therefore, they were excluded from the study. This suggests that identifying the anesthetic effect or upper sensory level before using a bolus of norepinephrine may reduce the occurrence of hypertension.

The current study further proves the effectiveness and safety of prophylactic use of norepinephrine in preventing hypotension after SA during CD. It also provides a new clinical idea of reducing the rate of hypotension, maintaining hemodynamic stability, and reducing the necessary dose of vasopressors by prophylactic use of a small bolus norepinephrine.

\section{Conclusions}

The $\mathrm{ED}_{90}$ of a norepinephrine bolus to prevent hypotension and as a rescue bolus for hypotension after CSE during CD was determined to be $10.85 \mu \mathrm{g}$ (95\% CI, 9.20$11.67 \mu \mathrm{g})$ and $12.3 \mu \mathrm{g}(95 \% \mathrm{CI}, 10.0-12.8 \mu \mathrm{g})$, respectively, using isotonic regression methods. A norepinephrine prophylactic bolus dose of $11 \mu \mathrm{g}$ or a rescue bolus dose of $12 \mu \mathrm{g}$ was recommended for clinical practice. Both methods appear to either prevent hypotension or act as a rescue medication for hypotension after CSE, and adverse outcomes were not observed. Furthermore, the prophylactic use of norepinephrine may have more advantages in the reduction of unpleasant symptoms related to hypotension, reducing the likelihood of using rescue drugs, and increasing oxygen supplementation for fetuses than when using a rescue bolus of norepinephrine.

\section{Acknowledgment}

The authors would like to acknowledge Professor Hui Zhang (from department of Biostatistics, St. Jude Children's Research Hospital, Memphis TN, USA) for his introduction Fang Wang to us.

\section{Footnote}

Conflicts of Interest: The authors have no conflicts of interest to declare.

Ethical Statement: The authors are accountable for all 
aspects of the work in ensuring that questions related to the accuracy or integrity of any part of the work are appropriately investigated and resolved. The present study obtained signed informed consent, and the research plan obtaining approval from the Research Ethics Committee of the International Peace Maternity and Child Health Hospital and registering at http://www.chictr.org.cn (INR1800016213).

\section{References}

1. Loubert C. Fluid and vasopressor management for Cesarean delivery under spinal anesthesia: continuing professional development. Can J Anaesth 2012;59:604-19.

2. Allen TK, George RB, White WD, et al. A doubleblind, placebo-controlled trial of four fixed rate infusion regimens of phenylephrine for hemodynamic support during spinal anesthesia for cesarean delivery. Anesth Analg 2010;111:1221-9.

3. Heesen M, Kölhr S, Rossaint R, et al. Prophylactic phenylephrine for caesarean section under spinal anaesthesia: systematic review and meta-analysis. Anaesthesia 2014;69:143-65.

4. Bishop DG, Cairns C, Grobbelaar M, et al. Prophylactic phenylephrine infusions to reduce severe spinal anesthesia hypotension during cesarean delivery in a resourceconstrained environment. Anesth Analg 2017;125:904-6.

5. Nag DS, Samaddar DP, Chatterjee A, et al. Vasopressors in obstetric anesthesia: a current perspective. World J Clin Cases 2015;3:58-64.

6. Ngan Kee WD, Khaw KS. Vasopressors in obstetrics: what should we be using? Curr Opin Anaesthesiol 2006;19:238-43.

7. Ngan Kee WD, Lee SW, Ng FF, et al. Randomized double-blinded comparison of norepinephrine and phenylephrine for maintenance of blood pressure during spinal anesthesia for cesarean delivery. Anesthesiology 2015;122:736-45.

Cite this article as: $\mathrm{Xu} \mathrm{T}$, Zheng J, An XH, Xu ZF, Wang F. Norepinephrine intravenous prophylactic bolus versus rescue bolus to prevent and treat maternal hypotension after combined spinal and epidural anesthesia during cesarean delivery: a sequential dose-finding study. Ann Transl Med 2019;7(18):451. doi: $10.21037 /$ atm.2019.08.50
8. Stewart A, Fernando R, McDonald S, et al. The dose-dependent effects of phenylephrine for elective cesarean delivery under spinal anesthesia. Anesth Analg 2010;111:1230-7.

9. Chen XZ, Chen H, Lou AF, et al. Dose-response study of spinal hyperbaric ropivacaine for cesarean section. J Zhejiang Univ Sci B 2006;7:992-7.

10. Stylianou M, Flournoy N. Dose finding using the biased coin up-and-down design and isotonic regression. Biometrics 2002;58:171-7.

11. Pace NL, Stylianou MP. Advances in and limitations of up-and-down methodology: a précis of clinical use, study design, and dose estimation in anesthesia research. Anesthesiology 2007;107:144-52.

12. Stylianou M, Proschan M, Flournoy N. Estimating the probability of toxicity at the target dose following an upand-down design. Stat Med 2003;22:535-43 .

13. Diciccio TJ, Efron B. Bootstrap confidence intervals. Stat Sci 1996;11:189-228.

14. Onwochei DN, Ngan Kee WD, Fung L, et al. Norepinephrine intermittent intravenous boluses to prevent hypotension during spinal anesthesia for cesarean delivery: a sequential allocation dose-finding study. Anesth Analg 2017;125:212-8.

15. Ngan Kee WD. A Random-allocation graded doseresponse study of norepinephrine and phenylephrine for treating hypotension during spinal anesthesia for cesarean delivery. Anesthesiology 2017;127:934-41.

16. Kyokong O, Charuluxananan S, Sriprajittichai P, et al. The incidence and risk factors of hypotension and bradycardia associated with spinal anesthesia. J Med Assoc Thai 2006;89 Suppl 3:S58-64.

17. Fakherpour A, Ghaem H, Fattahi Z, et al. Maternal and anaesthesia-related risk factors and incidence of spinal anaesthesia-induced hypotension in elective caesarean section: a multinomial logistic regression. Indian J Anaesth 2018;62:36-46. 